\title{
Decoking of Fixed-bed Catalytic Reactors
}

\author{
K. Roel Westerterp, H. Jan Fontein and Frits P.H. van Beckum* \\ Dedicated to Prof. Dr. Hanns Hofmann at the occasion of his 65th birthday
}

A mathematical model for the description of the non-steady state process of decoking of a fixed bed catalytic reactor is presented. The relevant dimensionless groups are identified and their influence on the process discussed. Appropriate relationships are given for the estimation of the maximum temperature in the bed. Methods of monitoring the process and of controlling it in the case of unknown or variable coke contents are explained.

\section{Introduction}

In catalytic hydrocarbon conversion processes, coke is generally deposited on the catalyst, thereby impairing its activity. Therefore, the catalyst must be periodically regenerated. This is effected by shutting the reactor down, removing the reaction mixture by purging with an inert gas and, if necessary, partly cooling down the catalyst bed and then passing an oxygenic gas through the reactor in order to burn off the coke. A non-steadystate, exothermic process occurs in the fixed bed reactor: a reaction zone migrates through the bed, the heat generated being transported by the gas and temporarily retained by the catalyst. In order to prevent deterioration of the catalyst, it is of the utmost importance that the temperature in the bed should not exceed a certain maximum permissible level.

Control of the regeneration is only possible if the highest temperature is recorded throughout the process; a scheme is presented for the positioning of thermocouples in such a way that this requirement is met. The scheme is based on relationships which describe the temperature profiles in the reactor and which are derived from the mathematical model of the regeneration process.

Previous studies, surveyed in [8], differ mainly in the treatment of the reaction kinetics and heat transfer. In the earliest publications $[1,2]$, a constant reaction rate was assumed during the process. Later, more realistic models were based on the diffusion of oxygen within the catalyst pellet $[4,6,7]$ or on an Arrhenius type exponential function of temperature [5]; in a recent article, the reaction is assumed to be controlled by mass transfer of oxygen from the gas to the catalyst surface [9]. With regard to heat transfer, some workers $[1,2,4,7]$ assume its rate to be infinite so that there is no difference in temperature between the gas and the catalyst bed. The heat transfer rate, however, is related to mass transfer rate by the Chilton-Colburn analogy or by the Lewis number; consequently, an infinite heat transfer rate would entail an infinite mass transfer rate. Thus, a more realistic approach would take both heat and mass transfer resistance into account.

\footnotetext{
* Prof. Dr. Ir. K.R. Westerterp, Ir. H.J. Fontein, and Drs. F.P.H. van Beckum, Dept. of Chemical Technology and Applied Mathematics, Twente University of Technology, P.O.Box 217, NL-7500 AE Enschede.
}

\section{Mathematical Model and its Solution}

In the present study, the reaction rate is assumed to be controlled by mass transfer which implies a finite heat transfer rate. Further assumptions are in accordance with those made by previous workers:

- the reactor is adiabatic,

- the flow of the gas is plug flow; density variations are negligible

- axial mass and heat dispersion is neglected,

- carbon deposits are completely burnt to $\mathrm{CO}_{2}$.

The assumption of mass transfer control for the burn-off rate leads to the highest rates which are possible. Reaction kinetics and pore diffusion can reduce only the rate of decoking and heat evolution, so that, if the reactor is operated according to the requirements based on this assumption, the maximum permissible temperature would not be exceeded. In addition, the assumption of a complete combustion to $\mathrm{CO}_{2}$ leads to the maximum possible heat evolution: the heat effect of partial combustion to $\mathrm{CO}$ is smaller and that of the combustion of chemically bound $\mathrm{H}$ is of the same order of magnitude as that of the complete combustion to $\mathrm{CO}_{2}$.

With these assumptions, the following balance equations are obtained:

$$
\begin{aligned}
& - \text { the oxygen }\left(\mathrm{O}_{2}\right) \text { mass balance: }{ }^{1)} \\
& \qquad \varepsilon \frac{\partial C_{\mathrm{O} 2}}{\partial t}=-u \frac{\partial C_{\mathrm{O} 2}}{\partial z}-k_{\mathrm{G}} a_{\mathrm{p}}(1-\varepsilon) C_{\mathrm{O} 2}
\end{aligned}
$$

- the coke (C) mass balance:

$$
(1-\varepsilon) \varrho_{\mathrm{s}} \frac{\partial C_{\mathrm{C}}}{\partial t}=-k_{\mathrm{G}} a_{\mathrm{p}}(1-\varepsilon) C_{\mathrm{O} 2} \text {, }
$$

- the gas heat balance:

$$
\varepsilon\left(\varrho C_{\mathrm{p}}\right)_{\mathrm{G}} \frac{\partial T}{\partial t}=-u\left(\varrho C_{\mathrm{p}}\right)_{\mathrm{G}} \frac{\partial T}{\partial z}+\alpha a_{\mathrm{p}}(1-\varepsilon)\left(T_{\mathrm{i}}-T\right)
$$

1) List of symbols at the end of the paper. 
- the solids heat balance:

$$
\begin{aligned}
(1-\varepsilon)\left(\varrho C_{\mathrm{p}}\right)_{\mathrm{S}} \frac{\partial T_{\mathrm{i}}}{\partial t}= & -\alpha a_{\mathrm{p}}(1-\varepsilon)\left(T_{\mathrm{i}}-T\right)+ \\
& +k_{\mathrm{G}} a_{\mathrm{p}}(1-\varepsilon) C_{\mathrm{O} 2} \Delta H
\end{aligned}
$$

where $T$ is the gas temperature and $T_{\mathrm{i}}$ that of the catalyst pellets, which are assumed to be isothermal.

The equations can be made dimensionless by introducing

$$
\begin{aligned}
& \tau=\frac{u t}{\varepsilon L}, \quad Z=\frac{z}{L}, \\
& \theta=\frac{T}{T_{0}}, \quad \theta_{\mathrm{i}}=\frac{T_{\mathrm{i}}}{T_{0}}, \\
& X_{\mathrm{O} 2}=\frac{C_{\mathrm{O} 2}}{C_{\mathrm{O} 2 \mathrm{o}}}, \quad X_{\mathrm{C}}=\frac{C_{\mathrm{C}}}{C_{\mathrm{Co}}} .
\end{aligned}
$$

The resulting equations are

$$
\begin{aligned}
& \frac{\partial X_{\mathrm{O} 2}}{\partial \tau}+\frac{\partial X_{\mathrm{O} 2}}{\partial Z}=-A X_{\mathrm{O} 2} \\
& \frac{\partial X_{\mathrm{C}}}{\partial \tau}=-A B X_{\mathrm{O} 2} \\
& \frac{\partial \theta}{\partial \tau}+\frac{\partial \theta}{\partial Z}=A C\left(\theta_{\mathrm{i}}-\theta\right) \\
& \frac{\partial \theta_{\mathrm{i}}}{\partial \tau}=-A C D\left(\theta_{\mathrm{i}}-\theta\right)+A B E X_{\mathrm{O} 2}
\end{aligned}
$$

The boundary conditions are

$$
\begin{array}{ll}
X_{\mathrm{O} 2}=0, X_{\mathrm{C}}=1 & \text { for } \tau \leqq Z, \\
X_{\mathrm{O} 2}=1 & \text { for } Z=0, \text { all } \tau>0, \\
\theta=\theta_{\mathrm{i}}=1 & \text { for } \tau=0,0 \leqq Z \leqq 1, \\
\theta=1 & \text { for } Z=0, \text { all } \tau>0 .
\end{array}
$$

This means that, after shutdown, the reactor is cooled to temperature $T_{\mathrm{o}}$ and decoking is started at $t=0$, with a regeneration gas containing $C_{\mathrm{O} 20} \mathrm{kmol}$ of oxygen per $\mathrm{m}^{3}$.

Eqs (6) through (9) contain five dimensionless groups, i.e.:

$$
\begin{aligned}
& A=\frac{k_{\mathrm{G}} a_{\mathrm{p}}(1-\varepsilon) L}{u}, \quad D=\frac{\varepsilon\left(\varrho C_{\mathrm{p}}\right)_{\mathrm{G}}}{(1-\varepsilon)\left(\varrho C_{\mathrm{p}}\right)_{\mathrm{S}}}, \\
& B=\frac{C_{\mathrm{O} 2} \varepsilon}{C_{\mathrm{Co}} \varrho_{\mathrm{S}}(1-\varepsilon)}, \quad E=\frac{\Delta H C_{\mathrm{Co}} \varrho_{\mathrm{S}}}{\left(\varrho C_{\mathrm{p}}\right)_{\mathrm{S}} T_{0}}, \\
& C=\frac{\alpha}{k_{\mathrm{G}}\left(\varrho C_{\mathrm{p}}\right)_{\mathrm{G}}} .
\end{aligned}
$$

. The significance of these groups is as follows:

- $A$ is the total number of mass transfer units in the reactor, while the height of a mass transfer unit is given by

$$
\mathrm{HTU}_{\mathrm{M}}=\frac{u}{k_{\mathrm{G}} a_{\mathrm{p}}(1-\varepsilon)} .
$$

$-B$ is the ratio of molar oxygen concentration in the feed gas to coke deposit originally present per unit reactor volume.

- $C$ is the ratio of heat to mass transfer per unit driving force and per unit particle surface area. On the basis of the Chilton-Colburn analogy $C \approx(\mathrm{Sc} / \mathrm{Pr})^{-0.67}=\mathrm{Le}^{-0.67}$ and therefore approximately equal to unity [10].

- $D$ is the ratio of gas to catalyst heat capacities per unit reactor volume.

- $E$ is the dimensionless adiabatic increase in temperature of the solid phase.

Many other dimensionless groups can also be formulated. Thus, e.g. $A C$ is the total number of heat transfer units in the reactor and $E B / D$ the dimensionless adiabatic increase in temperature of the gas phase.

The differential equations are conveniently solved by the wellknown method of characteristics [3] on introducing new independent variables:

$\phi=\tau-Z$ and $\psi=Z$.

The mass balances, which are independent of temperature, can be solved separately from the heat balances. With Eq. (15), they become

$$
\frac{\partial X_{\mathrm{O} 2}}{\partial \psi}=-A X_{\mathrm{C}}, \quad \frac{\partial X_{\mathrm{C}}}{\partial \phi}=-A B X_{\mathrm{O} 2} .
$$

Integration and substitution of the original independent variables yield:

$$
X_{\mathrm{O} 2}= \begin{cases}0 & \text { for } \tau<Z \\ \exp \left(-A\left(Z-Z_{\mathrm{rf}}\right)\right) & \text { for } \tau \geqq Z, Z \geqq Z_{\mathrm{rf}}(16) \\ 1 & \text { for } \tau \geqq Z, Z \leqq Z_{\mathrm{rf}}\end{cases}
$$$$
X_{\mathrm{C}}= \begin{cases}1 & \text { for } \tau<Z \\ 1-A B(\tau-Z) \exp (-A Z) & \text { for } \tau \geqq Z, \tau<\tau_{\mathrm{o}} \\ 1-\exp \left(-A\left(Z-Z_{\mathrm{r}}\right)\right) & \text { for } \tau \geqq Z, \tau>\tau_{\mathrm{o}}\end{cases}
$$

The heat balances can also be solved analytically. However, the resulting equations are rather complicated [9]: therefore, a numerical solution is preferred.

Reintroducing the variables $\phi$ and $\psi$, these balances are written as

$\frac{\partial \theta}{\partial \psi}=A C\left(\theta_{\mathrm{i}}-\theta\right)$ 
with $\zeta= \begin{cases}0 & \text { if } Z<Z_{\mathrm{rf}} \\ \exp \left(-A\left(Z-Z_{\mathrm{rf}}\right)\right) & \text { if } Z \geqq Z_{\mathrm{rf}}\end{cases}$

As indicated by Gonzalez and Spencer [5], the solutions of Eqs (18) can be approximated by the following difference equations:

$$
\begin{aligned}
\theta^{\phi, \psi+\Delta \psi}= & \theta_{\mathrm{i}}^{\phi, \psi}-\left(\theta_{\mathrm{i}}^{\phi, \psi}-\theta^{\phi, \psi}\right) \exp (-A C \Delta \psi) \\
\theta_{\mathrm{i}}^{\phi+\Delta \phi, \psi}= & \theta_{\mathrm{i}}^{\phi, \psi} \exp (-A C D \Delta \phi)+ \\
& +\left(\theta^{\phi, \psi}+\frac{E B \zeta}{C D}\right)(1-\exp (-A C D \Delta \phi))
\end{aligned}
$$

With the boundary conditions:

$\theta_{\mathrm{i}}=1 \quad$ for $\phi=0$ and $0 \leqq \psi \leqq 1$,

$\theta=1 \quad$ for $\phi=0$ and $0 \leqq \psi \leqq 1$, for $\psi=0$ and $\phi \geqq 0$.

The simultaneous partial differential equations can now be solved and, hence, temperature profiles are obtained as functions of time $\tau$ or of location $Z$. As an example, some temperature profiles shown in Fig. 2 will be discussed later in the text.

Depending on the choice of parameter values, an infinite number of profiles can be obtained. However, this is not sufficient for a real understanding of the process of air decoking; to that end, the real dimensionless groups which actually govern the process must be identified and also the way in which they influence the relevant phenomena. An attempt at this identification is made in the following section.

\section{Interpretation of the Calculated Profiles}

Unless stated otherwise, the basic data as given in Table 1 have been used for all the following calculations. They refer to the decoking of a catalyst bed, $8 \mathrm{~m}$ long, with a uniform coke deposit of 3.4 mass- $\%$, by a gas containing 1 vol.- $\%$ of oxygen. The initial temperature of the bed is $400{ }^{\circ} \mathrm{C}$ and the interstitial gas velocity $2.5 \mathrm{~m} / \mathrm{s}$, so that the gas residence time in the bed is $3.2 \mathrm{~s}$. The adiabatic increase in temperature, based on the solids phase, is $1060^{\circ} \mathrm{C}$.

\subsection{Velocity of the Reaction Front}

At the inlet cross-section of the reactor, $C_{\mathrm{O} 2}=C_{\mathrm{O} 20}$ for all $t>0$ : this means that the coke mass balance can be integrated for $Z=0$. It follows from this integration that the time needed to remove all the coke in the inlet cross-section is $\left(\varrho_{\mathrm{S}} C_{\mathrm{Co}}\right) /\left(k_{\mathrm{G}} a_{\mathrm{p}} C_{\mathrm{O} 2 \mathrm{o}}\right)$. After this induction period $t_{0}$, a "reaction front" moves through the reactor with a velocity $u_{\mathrm{rf}}$; if its position is given by $z_{\mathrm{rf}}$,

$$
\text { then } \begin{aligned}
C_{\mathrm{Co}}=0 & \text { for } z \leqq z_{\mathrm{rf}}, \\
C_{\mathrm{Co}}>0 & \text { for } z>z_{\mathrm{rf}},
\end{aligned}
$$

and

$$
z_{\mathrm{rf}}=u_{\mathrm{rf}}\left(t-t_{\mathrm{o}}\right) .
$$

Table 1. Data for the test case.

\begin{tabular}{lll}
\hline$a_{\mathrm{p}}$ & $=1200 \mathrm{~m}^{2} / \mathrm{m}^{3}$ \\
$\left(C_{\mathrm{p}}\right)_{\mathrm{G}}$ & $=1020 \mathrm{~J} / \mathrm{kgK}$ \\
$\left(C_{\mathrm{p}}\right)_{\mathrm{S}}$ & $=1050 \mathrm{~J} / \mathrm{kgK}$ \\
$\Delta H$ & $=394 \mathrm{MJ} / \mathrm{kmol}$ \\
$k_{\mathrm{G}}$ & $=0.15 \mathrm{~m} / \mathrm{s}$ \\
$L$ & $=8 \mathrm{~m}$ \\
$d_{\mathrm{p}}$ & $=0.005 \mathrm{~m}$ \\
$T_{\mathrm{o}}$ & $=673 \mathrm{~K}$ \\
$u$ & $=1 \mathrm{~m} / \mathrm{s}$ \\
$\alpha$ & $=77 \mathrm{~W} / \mathrm{m}^{2} \mathrm{~K}$ \\
$\varepsilon$ & $=0.4$ \\
$\varrho_{\mathrm{S}}$ & $=1400 \mathrm{~kg} / \mathrm{m}^{3}$ \\
$\varrho_{\mathrm{G}}$ & $=0.50 \mathrm{~kg} / \mathrm{m}^{3}$ \\
$\operatorname{Re}$ & $=173$ \\
With $C_{\mathrm{O} 2 \mathrm{o}}$ & $=0.18 \times 10^{-3} \mathrm{kmol} / \mathrm{m}^{3}\left(1\right.$ vol. \%) and $C_{\mathrm{Co}}=2.8 \times 10^{-3}$ \\
$\mathrm{kmol} / \mathrm{kg}(3.4 \mathrm{wt}-\%), \mathrm{the}^{-3}$ following values are obtained for the dimensionless \\
groups: & \\
$A$ & $=864$ \\
$B$ & $=30.3 \times 10^{-6}$ \\
$C$ & $=1.0$ \\
$D$ & $=230 \times 10^{-6}$ \\
$E$ & $=1.58$ \\
\hline
\end{tabular}

An expression for $u_{\mathrm{rf}}$ can be derived by assuming that the reaction front moves over a distance $\Delta z$ in the time interval $\Delta t$. During this interval, $C_{\mathrm{O} 2 \mathrm{o}} u S \Delta t$ moles of oxygen are fed to the reactor; this oxygen is used to burn off the coke in the volume element between coke profiles at times $t$ and $t+\Delta t$, see Fig. 1 and, subsequently, to fill this volume. Since, as had been shown, see Eq. (17), the coke profiles are independent of time, the two shaded areas in Fig. 1 are equal and thus

$$
C_{\mathrm{O} 2 \mathrm{o}} u S \Delta t=\left[\varepsilon C_{\mathrm{O} 2 \mathrm{o}}+(1-\varepsilon) \mathrm{e}_{\mathrm{s}} C_{\mathrm{Co}}\right] S \Delta z
$$

or

$$
u_{\mathrm{rf}}=\frac{\Delta z}{\Delta t}=\frac{u C_{\mathrm{O} 2 \mathrm{o}}}{(1-\varepsilon) \varrho_{\mathrm{s}} C_{\mathrm{Co}}+\varepsilon C_{\mathrm{O} 2 \mathrm{o}}}
$$

In dimensionless form

$\tau_{\mathrm{o}}=\frac{1}{A B}$,

$Z_{\mathrm{rf}}=\frac{B}{1+B}\left(\tau-\tau_{0}\right)$

or, since $B \ll 1$ :

$Z_{\mathrm{rf}}=B\left(\tau-\tau_{0}\right)$

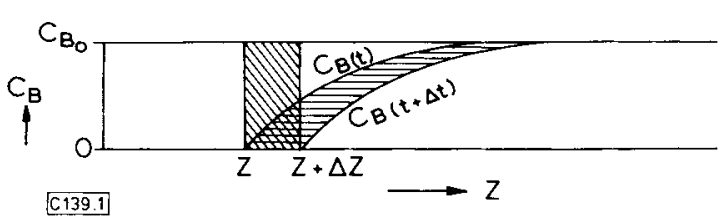

Fig. 1. Coke profiles at times $t$ and $t+\Delta t$. 
As a consequence, boundary condition (11) becomes

$X_{\mathrm{O} 2}=1 \quad$ for $Z \leqq Z_{\mathrm{rf}}$, all $\tau>0$

and, in addition,

$X_{\mathrm{C}}=0 \quad$ for $Z \leqq Z_{\mathrm{rf}}$, all $\tau \geqq \tau_{0}$.

Eq. (20) can be rewritten as

$U_{\mathrm{rf}}^{*}=u_{\mathrm{rf}} /(u / \varepsilon)=B$

since $U_{\mathrm{rf}}^{*}=Z_{\mathrm{rf}} / \tau$ and also $B \ll 1$. This now implies that the dimensionless group $B$ represents the dimensionless velocity of the reaction front.

Eqs (19) permit the computation of gas and catalyst temperature profiles throughout the reactor, for given values of $\phi[5]$. It follows from Eqs (20) and (21) that regeneration is complete at time $\tau_{\mathrm{e}}=1 / B+\tau_{0}$. The values of $\tau_{0}$ and $B$ are of the order of 10 and $10^{-4}$, respectively, whereas $Z$ is always smaller than 1. Therefore $\tau \gg Z$ applies during practically the whole process of regeneration, so that the obtained profiles can be regarded as a very good approximation of those at given values of $\tau$.

Some profiles are shown in Fig. 2. The dimensionless groups were computed from the values of the physical constants given in Table 1. These profiles indicate that there is an expanding zone in the reactor where reaction heat accumulates. After an initial period, gas and catalyst temperatures remain constant and virtually the same in the larger part of the accumulation zone. This constant temperature, $\theta_{i, p}$, is independent of time. The initial period is longer, the higher the value of $B$; constant $\theta_{\mathrm{i}}$ has not yet been reached in Figs $2 b$ and $2 c$. With regard to Fig. 2a, the heating up of the catalyst mass as a result of combustion, i.e. the excess temperature $\theta_{i}-1$, is relatively moderate, in the present example $160^{\circ} \mathrm{C}$ but, in Figs $2 \mathrm{~b}$ and $2 \mathrm{c}$, it becomes excessive, namely 1825 and $4500^{\circ} \mathrm{C}$, respectively. It is evident that in the last two cases the temperature rises would be so extreme that the catalyst mass and the reactor would be destroyed. Therefore, the temperature control during decoking is extremely important. The burn-off of the coke in the inlet cross-section for the test case of Table 1 takes $122 \mathrm{~s}$. The reaction front moves at a velocity of $0.076 \mathrm{~mm} / \mathrm{s}$ and the burn-off takes $29 \mathrm{~h} 20 \mathrm{~min}$.

\subsection{Length of the Reaction Zone}

The coke profile in the catalyst bed follows from Eq. (17). Defining the reaction zone as the bed length from the location where all the coke has just been burnt off, or $Z=Z_{\text {rf }}$, to that where just the first percent of coke has been burnt off, here $X_{\mathrm{C}}$ $=0.01$ or $Z-Z_{\mathrm{rf}}=4.6 / \mathrm{A}$, the length of the reaction zone is given by

$L_{\mathrm{rf}}=4.6 L / A$.

In the present example, the length of the reaction zone is $43 \mathrm{~mm}$ or equivalent to 9 catalyst particles. This value can also be obtained by the method outlined in [10]. It should be realized that

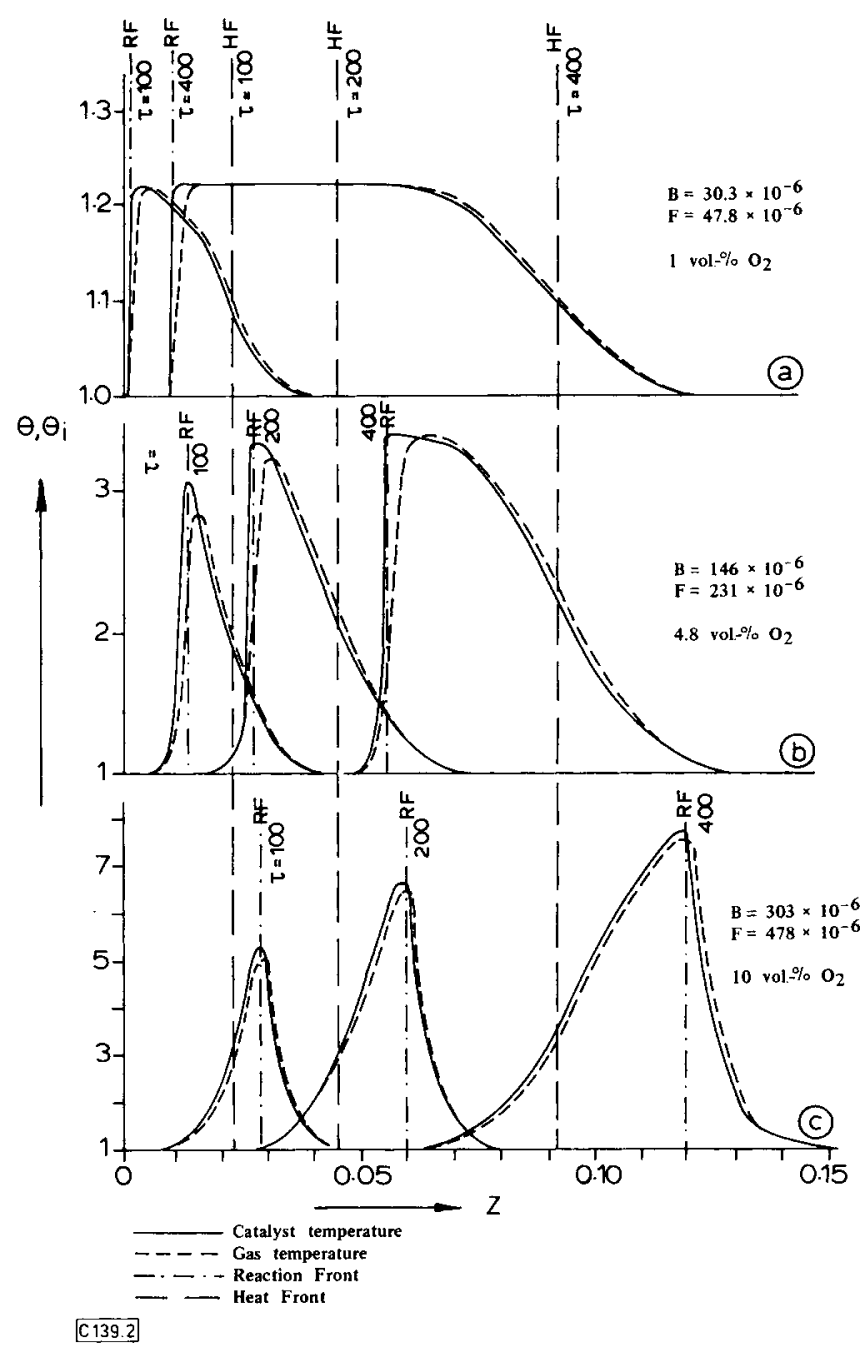

Fig. 2. Temperature profiles: a) test case with 1 vol. $-\% \mathrm{O}_{2}$ and $D / B=7.6$, b) 4.8 vol. $\% \mathrm{O}_{2}$ and $D / B=1.58$, c) $10 \mathrm{vol}$. $\%$ and $D / B=0.76$.

the reaction zone is extremely short and, for this reason, very difficult to detect during decoking of a catalyst bed.

\subsection{Velocity of the Heat Wave through the Bed}

The accumulation zone is bounded on one side by the reaction front at $Z_{\mathrm{rf}}$ and on the other side by a "heat front" at $Z_{\mathrm{hf}}$. Whereas, in Figs $2 \mathrm{a}$ and $2 \mathrm{~b}, Z_{\mathrm{rf}}<Z_{\mathrm{hf}}$, in Fig. 2c, $Z_{\mathrm{hf}}<Z_{\mathrm{rf}}$.

Heat is transported through the bed by the gas; therefore, if the heat front moves over the distance $\Delta z$ in time $\Delta t$, in analogy to the derivation of the reaction front velocity, $\left(C_{\mathrm{p}} \mathrm{e}\right)_{\mathrm{G}}\left(T-T_{0}\right) u \Delta t$ $=\left\{\varepsilon\left(C_{\mathrm{p}} \varrho\right)_{\mathrm{G}}\left(T-T_{0}\right)+(1-\varepsilon)\left(C_{\mathrm{p}} \varrho\right)_{\mathrm{S}}\left(T_{\mathrm{i}}-T_{0}\right\} \Delta z\right.$ or, since $T$ and $T_{\mathrm{i}}$ are approximately equal,

$u_{\mathrm{hf}}=\frac{\Delta z}{\Delta t}=\frac{\left(C_{\mathrm{p}} \varrho\right)_{\mathrm{G}} u}{\varepsilon\left(C_{\mathrm{p}} \varrho\right)_{\mathrm{G}}+(1-\varepsilon)\left(C_{\mathrm{p}} \varrho\right)_{\mathrm{S}}}$.

The first term in the denominator is very small in comparison to the second term, so that in dimensionless form: 
$Z_{\mathrm{hf}}=\frac{D}{1+D} \tau \approx D_{\tau}$

For the profiles in Figs $2 \mathrm{a}$ and $2 \mathrm{~b}$, the value of $B$ is lower than that of $D$; consequently $Z_{\mathrm{rf}}<Z_{\mathrm{hf}}$ and expansion of the accumulation zone occurs because the heat front migrates faster than the reaction front: the heat transported by the gas is given up to the catalyst at the heat front.

For the profiles in Fig. $2 \mathrm{c}$ on the other hand, $B>D$ and thus $Z_{\mathrm{rf}}>Z_{\mathrm{hf}}$. In this case, the reaction front moves faster than the heat front and the heat accumulates in the wake of the reaction front.

It is clear from looking at the profiles that, as already mentioned, the temperatures reached in Figs $2 b$ and $2 c$ are so high that the catalyst will be definitely damaged, and that only conditions such as those of Fig. 2 a are acceptable for decoking.

Now, the significance of the dimensionless group $D$ has also become clear, i.e. $D$ represents the dimensionless velocity of the heat front through the bed:

$U_{\mathrm{hf}}^{*}=D$

For the basic data in Table 1 , the velocity of the heat front is $575 \times 10^{-6} \mathrm{~m} / \mathrm{s}$, which means that it takes $3 \mathrm{~h} 52 \mathrm{~min}$ to reach the outlet of the catalyst bed.

\subsection{Temperature Plateau in the Catalyst Bed}

As demonstrated in Fig. 2a for moderate temperatures, a temperature plateau $\theta_{i, p}$ develops. An expression, which correlates $\theta_{i, p}$ to the process conditions, can be derived as follows. As long as all the heat generated still remains in the bed, the following balance equation is valid:

$$
\begin{aligned}
C_{\mathrm{O} 2 \mathrm{o}} \Delta H u t= & \varepsilon\left(\varrho C_{\mathrm{p}}\right)_{\mathrm{G}} \int_{\mathrm{z}=0}^{z=L}\left(T-T_{0}\right) \mathrm{d} z+ \\
& +(1-\varepsilon)\left(\varrho C_{\mathrm{p}}\right)_{\mathrm{S}} \int_{z=0}^{z=L}\left(T_{\mathrm{i}}-T_{0}\right) \mathrm{d} z
\end{aligned}
$$

or

$$
E B \tau \approx \int_{Z=0}^{Z=1}\left[D(\theta-1)+\left(\theta_{\mathrm{i}}-1\right)\right] \mathrm{d} Z,
$$

since $\theta \approx \theta_{\mathrm{i}}$ and $D \ll 1$,

$$
E B \tau \approx \int_{Z=0}^{Z=1}\left(\theta_{i}-1\right) \mathrm{d} Z \text {. }
$$

Assuming that the two shaded areas in Fig. 3 are equal,

$$
E B \tau \approx\left(\theta_{\mathrm{i}, \mathrm{p}}-1\right)\left(Z_{\mathrm{hf}}-Z_{\mathrm{rf}}\right)
$$

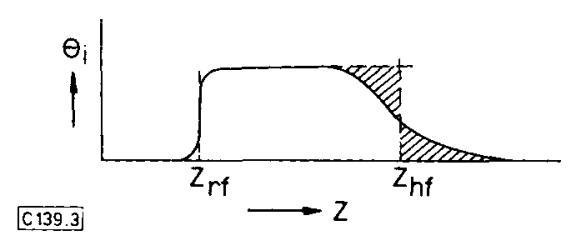

Fig. 3. Instant temperature profile in the catalyst.

or, for $\tau \gg \tau_{0}$

$\theta_{\mathrm{i}, \mathrm{p}}=1+\left|\frac{E}{(D / B)-1}\right|$.

It follows from Eq. (24) that if the two fronts move at the same velocity, i.e. if $D=B, \theta_{\mathrm{i}, \mathrm{p}}$ tends to infinity. This was observed by nearly all the previous investigators.

For the case of Table 1 , a plateau temperature of $560^{\circ} \mathrm{C}$ was obtained.

The difference between the plateau temperature $T_{\mathrm{i}, \mathrm{p}}$ and the initial bed temperature $T_{0}$ is equal to the adiabatic temperature increase of the solid phase $\Delta T_{\text {ads }}=T_{\mathrm{o}} E$, divided by $(D / B)-1$. For a low coke deposit, of say 1 mass- $\%$, the adiabatic temperature increase already approaches $300^{\circ} \mathrm{C}$. This implies that, in practice, the temperature increase of the bed must be kept below $\Delta T_{\text {ads }}$, in order to protect the catalyst; this can be achieved in the case $D / B=C_{\mathrm{Co}}\left(\varrho c_{\mathrm{p}}\right)_{\mathrm{G}} / C_{\mathrm{O} 2 \mathrm{o}} C_{\mathrm{pS}}>2$. In the test case of Table $1, D / B=7.6$.

It is seen that the temperature increase of the catalyst bed can be even much higher than $\Delta T_{\text {ads }}$, when $D / B<2$. In this case, the reaction front moves too fast in relation to the heat front and all the heat evolved has to be temporarily stored in a part of the bed which is too shallow.

\subsection{Over- or Underheating of the Reaction Zone}

A consequence of the values of the physical constants as given in Table 1 is that $C=1.00$. In order to study the influence of $C$, the ratio of heat to mass transfer, profiles were computed for values of $C$ in the range from 0.7 to 1.2 by adapting the value of the heat transfer coefficient. Fig. 4 gives some representative results. From these, it may be concluded that the profiles depend on $C$ solely in the reaction zone whose length is only about 10 to 20 particle diameters [10]. If $C<1$, the catalyst temperature passes through a peak at the reaction front because the heat transfer is slower than the mass transfer; the height of the peak depends on their ratio. On the other hand, if $C>1$ or if the heat transfer is faster than the mass transfer, the catalyst temperature at the reaction front lags behind to attain its maximum value only downstream from this front.

Like $\theta_{\mathrm{i}, \mathrm{p}}, \theta_{\mathrm{i}, \mathrm{r}}$, the catalyst temperature at the front, also becomes independent of time after the initial period. This period is the longer, the higher the oxygen content of the gas. 


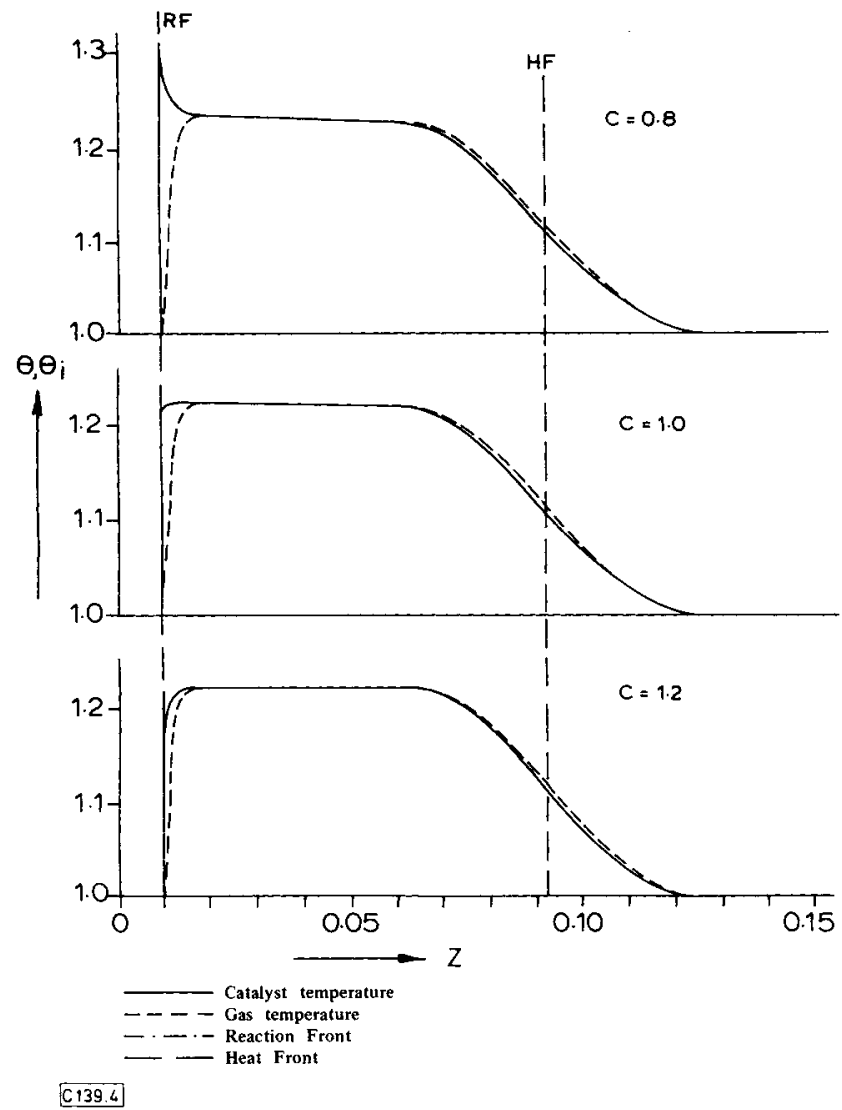

Fig. 4. Temperature profiles with over- and underheating of the reaction zone.

As shown in the appendix, the following expression can be derived for $\theta_{\mathrm{i}, \mathrm{rf}}$ from the heat balances:

$\theta_{\mathrm{i}, \mathrm{rf}}=1+\frac{E}{(D / B)-1} \frac{D / B}{1+C[(D / B)-1]}$.

It is shown in the appendix that the temperature of the reaction front is higher than the plateau temperature in case $C<1$.

$C$ can be estimated in the usual mixtures of gas and inert diluent for decoking. If steam is used as the inert diluent for air, at $300 \mathrm{~K}, C=0.89$ and, at $700 \mathrm{~K}$, it is $=0.80$ and, when nitrogen is used, $C=0.98$ at $300 \mathrm{~K}$ and around 0.84 at 700 $\mathrm{K}$. For the present test case, this leads to a maximum temperature in the reaction zone of $T_{i, \mathrm{rf}}=850 \mathrm{~K}$ for $C=0.9$ and of $869 \mathrm{~K}$ for $C=0.8$ or the maximum overheating in the reaction zone amounts to 17 and $36^{\circ} \mathrm{C}$ for the two cases.

The above leads to the conclusion that $\theta_{\mathrm{i}, \max }$, the maximum temperature of the catalyst bed during regeneration, is given by

$$
1+\left|\frac{E}{(D / B)-1}\right|
$$

if $C \geqq 1$,

$\theta_{\mathrm{i}, \max }=$

$$
1+\left|\frac{E}{(D / B)-1}\right| \frac{(D / B)}{1+C[(D / B)-1]} \quad \text { if } C<1 .
$$
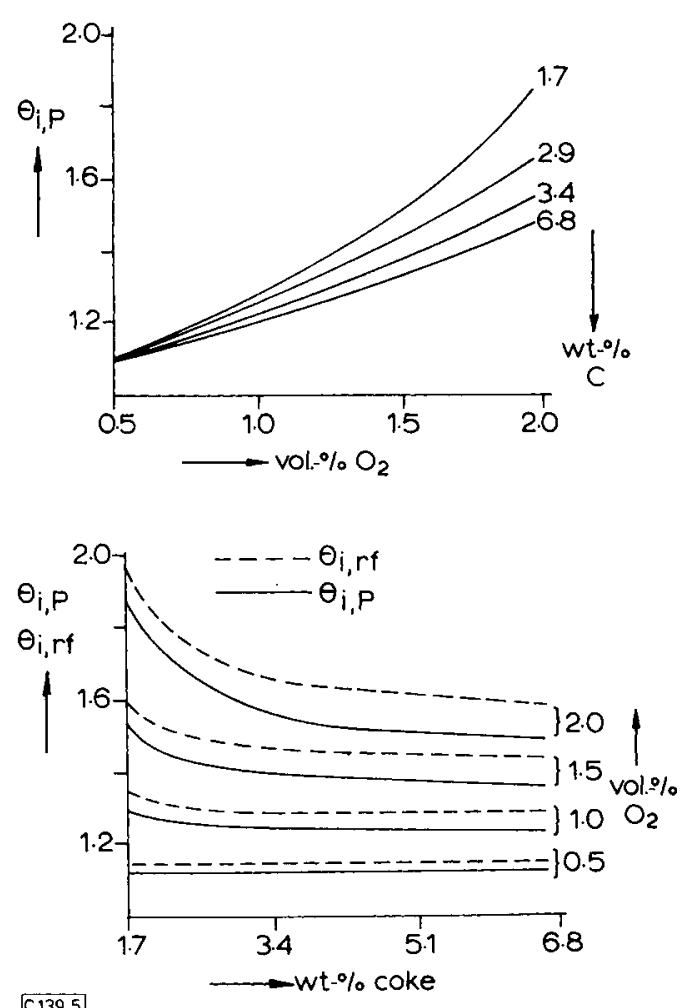

$C 139.5$

Fig. 5. Plateau and reaction front temperatures for $C=0.8$ as functions of coke content and oxygen concentration.

Fig. 5 presents $\theta_{i, \max }$ as a function of the oxygen content of the gas and coke concentration of the catalyst for $C=1$ and $C=$ 0.8 .

\section{Temperature Control}

Thermocouples are installed along the reactor in order to control its temperature during regeneration. It is imperative that at least one thermocouple should be in the accumulation zone throughout the process. Since this zone is bounded by the reaction and heat fronts, the positions $z_{1}, z_{2} \ldots$ of the thermocouples are determined by the construction shown in Fig. 6. In Fig. 6, the locations of the fronts are given at a certain time $\tau$. For moderate temperature increases, $D / B$ is much larger than unity

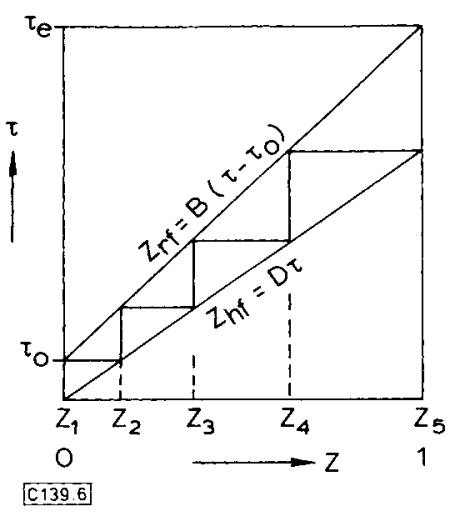

Fig. 6. Positioning of thermocouples. 
so that a relatively small number of thermocouples, above all near the inlet, must be installed. This implies that the values of $B$ and $D$ have to be estimated in the design phase. It can be seen from their definitions, Eq. (14), that $D$ depends only on physical constants, in contrast to $B$, which also depends on the ratio of the oxygen concentration $C_{\mathrm{O} 2 \mathrm{o}}$ of the gas to the coke content $C_{\mathrm{Co}}$ of the catalyst. It is apparent from Fig. 6 that at higher values of $B$, the thermocouples should be spaced at smaller intervals. Therefore, for a safe design, the smallest coke content still expected to require regeneration of the catalyst, as preferably determined in the development laboratory, should be adopted for $C_{\mathrm{Co}}$; for $C_{\mathrm{O} 20}$ the highest oxygen concentration for which the estimate of $\theta_{i, \max }$ to be obtained with Eq. (26) does not exceed the maximum permissible catalyst temperature.

Actual operation of a regeneration process should start with the oxygen concentration on which the design is based. If the coke content of the bed exceeds the design value, the bed temperature read from a thermocouple will be lower than the permitted temperature and the oxygen partial pressure can be increased accordingly to speed up the process. However, it should be borne in mind that if the Lewis number is larger than 1 , the temperature indicated by the thermocouples is not the maximum temperature $\theta_{i, \text { f }}$, but only $\theta_{i, p}$, unless the reaction front passes through the location of one of the thermocouples. The Lewis number depends on the composition of the regeneration gas to be used and has to be determined beforehand. Furthermore, as can be seen from Fig. 3, in the location of the heat front, the plateau temperature has not yet been reached; hence, this also requires a slightly more dense thermocouple pattern. For the test case, the thermocouples have to be inserted in the inlet cross-section and at distances of $0.07,0.53$ and $4.01 \mathrm{~m}$ from the inlet and also in the outlet of the reactor. In the extreme case of a very low coke content, with e.g. $B=115 \times 10^{-6}$ and when $D / B=2$, the thermocouples would have to be located in the inlet cross-section and at distances of $0.02,0.06,0.13$, $0.28,0.57,1.16,2.33$ and $4.67 \mathrm{~m}$ from the inlet.

The carbon content in the bed is often unknown. It is also possible for the carbon content to change along the bed, e.g. to decrease in the direction of the flow. In this case, $E, D / B$ and $B$ are unknown. It is clear from Eq. (24) that a reduction in the coke content increaes the plateau temperature in the bed. In such situations, the process is started with a low oxygen content in the gaseous feed which is gradually and carefully increased according to the bed temperature. By measuring the time required for the passage of the reaction front between two thermocouples, the coke content in the bed between these two thermocouples can be determined even empirically. A decrease in the coke content must be compensated for by a decrease of the oxygen content in the feed gas, thus maintaining $T_{\mathrm{i}, \max }$ below the maximum permitted catalyst temperature. Additional thermocouples have to be inserted into the bed if the coke content gradually decreases in the axial direction.

\section{Conclusions}

The process of decoking has been described and explained. The process is governed by five dimensionless groups:
- $k_{\mathrm{G}} a_{\mathrm{p}}(1-\varepsilon) L / u$ is the total number of mass transfer units in the bed. It mainly determines the length of the reaction zone which is usually very short.

- $\Delta H C_{\mathrm{Co}} / C_{\mathrm{ps}} T_{0}$ is the dimensionless adiabatic increase in temperature of the solid phase.

$-C_{\mathrm{O} 2 \mathrm{o}} \varepsilon / C_{\mathrm{Co}_{0}} \varrho_{\mathrm{s}}(1-\varepsilon)$ is the dimensionless velocity of the reaction front.

$-\left(\varrho C_{\mathrm{p}}\right)_{\mathrm{G}} \varepsilon /\left(\varrho C_{\mathrm{p}}\right)_{\mathrm{S}}(1-\varepsilon)$ is the dimensionless velocity of the heat front.

$-\alpha / k_{\mathrm{G}}\left(\varrho C_{\mathrm{p}}\right)_{\mathrm{G}}$ determines the overheating or underheating of the reaction zone with respect to the plateau temperature.

Many other dimensionless groups can be formed but none of them has a direct bearing on only one single aspect of the decoking process.

In practice, the air decoking process is often conducted too slowly, for fear of overheating the catalyst. The described analysis provides a tool for monitoring the process and determining experimentally how to perform the decoking rapidly but safely.

\section{Symbols used}

\begin{tabular}{|c|c|c|}
\hline$A$ & {$[-]$} & $k_{\mathrm{G}} a_{\mathrm{p}}(1-\varepsilon) L / u$ \\
\hline$a_{\mathrm{p}}$ & {$\left[\mathrm{m}^{2} / \mathrm{m}^{3}\right.$ particle $]$} & particle surface area per unit volume \\
\hline$B$ & {$[-]$} & $C_{\mathrm{O} 2 \mathrm{o}} \varepsilon / C_{\mathrm{Co}_{\mathrm{S}}} \varrho_{\mathrm{S}}(1-\varepsilon)$ \\
\hline$C$ & {$[-]$} & $\alpha / k_{\mathrm{G}}\left(\varrho C_{\mathrm{p}}\right)_{\mathrm{G}}$ \\
\hline$C_{\mathrm{O} 2}$ & {$\left[\mathrm{kmol} / \mathrm{m}^{3}\right.$ gas $]$} & oxygen concentration \\
\hline$C_{\mathrm{C}}$ & [ $\mathrm{kmol} / \mathrm{kg}$ catalyst] & coke concentration \\
\hline$C_{\mathrm{p}}$ & {$[\mathrm{J} / \mathrm{kg} \mathrm{K}]$} & specific heat capacity \\
\hline$D$ & {$[-]$} & $\varepsilon\left(\varrho C_{\mathrm{p}}\right)_{\mathrm{G}} /(1-\varepsilon)\left(\varrho C_{\mathrm{p}}\right)_{\mathrm{S}}$ \\
\hline$E$ & {$[-]$} & $\Delta H C_{\mathrm{Co}} / C_{\mathrm{ps}} T_{\mathrm{o}}$ \\
\hline$F$ & {$[-]$} & $E B$ \\
\hline$\Delta H$ & {$[\mathrm{~J} / \mathrm{kmol}]$} & heat of reaction \\
\hline$k_{\mathrm{G}}$ & {$[\mathrm{m} / \mathrm{s}]$} & mass transfer coefficient \\
\hline$L$ & {$[\mathrm{~m}]$} & reactor length \\
\hline$S$ & {$\left[\mathrm{~m}^{2}\right]$} & reactor cross-sectional area \\
\hline$t$ & {$[\mathrm{~s}]$} & time \\
\hline$T$ & {$[\mathrm{~K}]$} & gas temperature \\
\hline$T_{\mathrm{i}}$ & {$[\mathrm{K}]$} & catalyst temperature \\
\hline$\Delta \mathrm{T}_{\mathrm{ad}}$ & {$[\mathrm{K}]$} & adiabatic temperature rise \\
\hline$u$ & {$[\mathrm{~m} / \mathrm{s}]$} & velocity \\
\hline$U_{\mathrm{i}}^{*}$ & {$[-]$} & $u_{\mathrm{i}} /(u / \varepsilon)$ \\
\hline$X$ & {$[-]$} & $C_{\mathrm{i}} / C_{\mathrm{io}}$, dimensionless concentration \\
\hline$z$ & {$[\mathrm{~m}]$} & length coordinate in reactor \\
\hline$Z$ & {$[-]$} & dimensionless length coordinate, $z / L$ \\
\hline$\alpha$ & {$\left[\mathrm{W} / \mathrm{m}^{2} \mathrm{~K}\right]$} & heat transfer coefficient \\
\hline$\varepsilon$ & {$[-]$} & bed porosity \\
\hline$\zeta$ & {$[-]$} & parameter, see Eq. (18) \\
\hline$\theta$ & {$[-]$} & dimensionless temperature, $T / T_{\mathrm{o}}$ \\
\hline e & {$\left[\mathrm{kg} / \mathrm{m}^{3}\right]$} & density \\
\hline$\tau$ & {$[-]$} & dimensionless time, $t u / \varepsilon L$ \\
\hline$\phi, \psi$ & {$[-]$} & characteristic coordinates \\
\hline
\end{tabular}

Indices

G gas

hf heat front

0 initial

P plateau

rf reaction front

S catalyst bed 


\section{References}

[1] van Deemter, J.J., Ind. Eng Chem. 45 (1953) No. 6., pp. $1227-1233$.

[2] van Deemter, J.J., Ind. Eng Chem. 46 (1954) No.11, pp. $2300-2302$.

[3] Acrivos, A., Ind. Eng Chem. 48 (1956) No. 4, pp. 703-710.

[4] Johnson, B.M., Froment, G.F., Watson, C.C., Chem. Eng Sci. 17 (1962) pp. 835-848.

[5] Gonzalez, L.O., Spencer, E.H., Chem. Eng Sci. 18 (1963) pp. $753-766$.

[6] Olson, K.E., Luss, O., Amundson, N.R., Ind. Eng Chem. Process Des. Dev. 7 (1968) No. 1, pp. $96-100$.

[7] Ozawa, Y., Ind. Eng Chem. Process Des. Dev. 8 (1969) No. 3, pp. $378-383$.

[8] Sampath, B.S., Hughes, R., Process Technol. Int. 18(1973) No. 1/2, pp. $39-43$.

[9] Marivoet, J., Wajc, S.J., Chem. Eng Sci. 32 (1977) pp. $779-782$.

[10] Westerterp, K.R., van Swaaij, W.P.M., Beenackers, A.A.C.M., Chemical Reactor Design and Operation, Wiley \& Sons, New York 1984.

\section{Appendix}

\section{Derivation of Eq. (25)}

In order to study the temperature distribution along the front, Eqs (8) and (9) are transformed to new coordinates $\sigma=\tau$ and $\xi=A C\left(Z-Z_{\mathrm{f}}\right)=$ $A C\left(Z-B\left(\tau-\tau_{\mathrm{o}}\right)\right)$ so that, at all times, the front position is given by $\xi=$ 0 , Fig. 7. The differential equations become:

$$
\begin{aligned}
& \frac{\partial \theta}{\partial \sigma}+A C(1-B) \frac{\partial \theta}{\partial \xi}=A C\left(\theta_{\mathrm{i}}-\theta\right), \frac{\partial \theta_{\mathrm{i}}}{\partial \sigma}-A B C \frac{\partial \theta_{\mathrm{i}}}{\partial \xi}= \\
& =-A C D\left(\theta_{\mathrm{i}}-\theta\right)+A F \zeta
\end{aligned}
$$

with $\zeta$, as in Eq. (18):

$$
\zeta\left\{\begin{array}{l}
0 \text { if } \xi<0 \\
\exp (-\xi / C) \text { if } \xi \geqq 0
\end{array}\right.
$$

After a start-up period, the temperature distribution in the vicinity of the front becomes independent of the time coordinate $\sigma$; its behaviour as a function of $\xi$ can be found by omitting the derivatives with respect to $\sigma$. Neglecting $B$ in the first equation, assuming $B<1$, leads to the system of ordinary differential equations:

$$
\frac{\mathrm{d}}{\mathrm{d} \xi}\left[\begin{array}{l}
\theta \\
\theta_{\mathrm{i}}
\end{array}\right]=\left[\begin{array}{ll}
-1 & 1 \\
-D / B & D / B
\end{array}\right]\left[\begin{array}{l}
\theta \\
\theta_{\mathrm{i}}
\end{array}\right]+\left[\begin{array}{l}
0 \\
-F \zeta / B C
\end{array}\right] .
$$

A standard solution technique leads to:

$$
\begin{aligned}
\xi<0:\left[\begin{array}{c}
\theta \\
\theta_{\mathrm{i}}
\end{array}\right]= & a\left[\begin{array}{l}
1 \\
1
\end{array}\right]+b\left[\begin{array}{l}
B \\
D
\end{array}\right] \mathrm{e}^{\lambda \xi}, \lambda=\frac{D}{B}-1, \\
\xi \geqq 0:\left[\begin{array}{c}
\theta \\
\theta_{\mathrm{i}}
\end{array}\right]= & \left(a-\frac{F}{B-D}\left(1-\mathrm{e}^{-\xi / C}\right)\right)\left[\begin{array}{l}
1 \\
1
\end{array}\right]+ \\
& +\left(b \mathrm{e}^{\lambda \xi}+\frac{F}{(B-D) B C} \frac{e^{\lambda \xi}-e^{-\xi / C}}{\lambda+1 / C}\right)\left[\begin{array}{l}
B \\
D
\end{array}\right],
\end{aligned}
$$

where $D \neq B(\lambda \neq 0)$ is assumed, and continuity throughout the front accounted for. The coefficients $a$ and $b$ are to be chosen in such a way that the temperature satisfies certain asymptotic boundary conditions. To this end, distinction is made between $\lambda>0$ and $\lambda<0$.

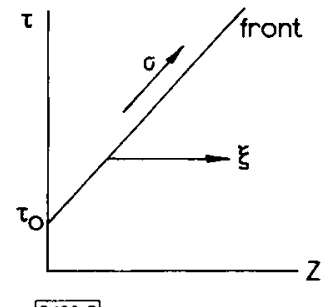

C 139.7

Fig. 7. Transformation of coordinates.

1) In the case $\lambda>0(D>B)$, the following is obtained from (A1) with $\xi \rightarrow \infty: a=1$, and from Eq. (A2) where the terms with $\mathrm{e}^{\lambda \xi}$ must cancel:

$b=F /(D-B) /(B+C(D-B))$. The behaviour of $\theta_{\mathrm{i}}$ is now given by:

$$
\begin{aligned}
\xi<0: \theta_{\mathrm{i}}= & 1+\frac{F}{D-B} \frac{D}{B+C(D-B)} \mathrm{e}^{\lambda \xi}, \\
\xi=0: \theta_{\mathrm{i}}=1+ & \frac{F}{D-B} \frac{D}{B+C(D-B)}=\theta_{\mathrm{i}, \mathrm{rf}} \text { c.f. Eq. (25) }, \\
\xi>0: \theta_{\mathrm{i}}= & 1+\frac{F}{D-B}+\frac{(1-C) F}{B+C(D-B)} \mathrm{e}^{-\xi / C}=\theta_{\mathrm{i}, \mathrm{rf}}+ \\
& +\frac{(C-1) F}{B+C(D-B)}\left(1-\mathrm{e}^{-\xi / C}\right) .
\end{aligned}
$$

For $\xi \rightarrow \infty$, the temperature $\theta_{\mathrm{i}}$ settles on the value $1+F /(D-B)$, cf. $\theta_{\mathrm{ip}}$ given by Eq. (24). This value may be higher, equal or lower than $\theta_{\mathrm{i}, \mathrm{r}}$, corresponding to whether $C-1$ is positive, zero or negative, see Fig. 8 .
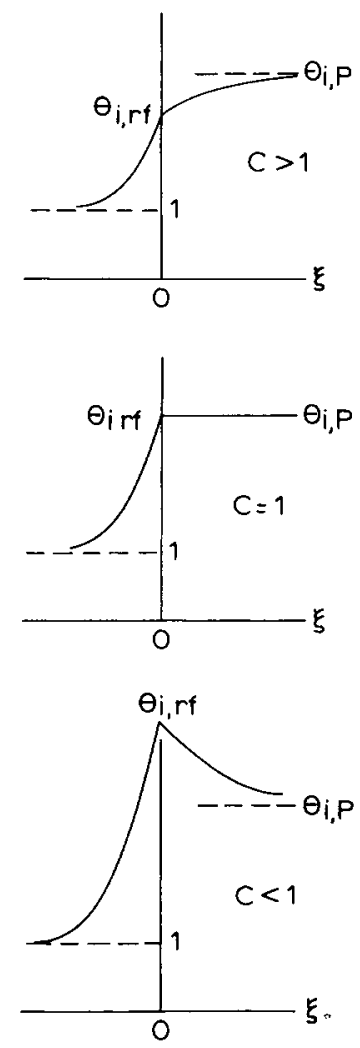

C139.8

Fig. 8. Possible temperature profiles near the reaction front for $D / B>1$. 
2) In the case $\lambda<0(D<B)$, the exponential term in Eq. (A1) must be suppressed, so that $b=0$; from Eq. (A2) with $\theta_{\mathrm{i}} \rightarrow 1$ for $\xi \rightarrow \infty$ we find $a=1+F /(B-D)$. Thus, see Fig. 9 :

$$
\begin{aligned}
& \xi \leqq 0: \theta_{\mathrm{i}}=a=1+F /(B-D)=\theta_{\mathrm{i}, \mathrm{rf}} \text { c.f. Eq. (24) } \\
& \xi>0: \theta_{\mathrm{i}} \text { decreases monotonously to } \theta_{\mathrm{i}}=1
\end{aligned}
$$

Here, the parameter $C$ does not play such a significant role as in the previous case. Only the special value $C=-1 / \lambda=B(B-D)$ will change the mathematical form of the solution (A2), but this does not affect the characteristic temperature behaviour.

3) For the sake of completeness, it should be observed that the case $\lambda=$ $0(D=B)$ is not suited to this treatment, because the continuous ac-

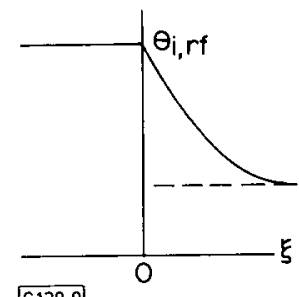

C139.9
Fig. 9. Temperature profile near the reaction front for $D / B<1$. cumulation of heat within the reaction zone prevents the temperature from reaching a $\sigma$-independent behaviour.

Received: March 28, 1988 [CET 139]

\title{
Model Discrimination and Parameter Estimation in the Kinetic Study of Methanol Synthesis
}

\author{
Jianguo Xu, Thomas Rickert and Ulrich Hoffmann* \\ Dedicated to Professor Dr. Hanns Hofmann on the occasion of his 65th birthday
}

The new model discrimination method is based on the correlation coefficient test of experimental data sets for different reactions, without requiring a prior parameter estimation. The new parameter estimation method reduces by $n$ the number of dimensions to be tested, compared to the classical method where $n$ is the number of independent reactions in the system. This has reduced the computation time for most complex reactions to a level, comparable to that needed for single reactions. The example used is the kinetic study of methanol synthesis in which the formation rates of methanol, methane, ethanol and ethane are considered. Two adequately accurate models were obtained from an extensive range of plausible models by using the new model discrimination and parameter estimation methods with a relatively small computation effort.

\section{Introduction}

Kinetic study of complex reactions has been a difficult task for two reasons. One is that the number of plausible models is very large, since the combination of different plausible rate equations for different reactions generates a large number of rate equation sets. For example, if four reactions are involved in a system, and for each product there are 10 possible rate equations depending on possible reaction paths and rate determining steps, the total number of plausible rate equation sets is as large as 10000 . The other reason is that the parameter estimation by the classical method [1] of minimizing the sum of residual squares or the more fundamental objective function $G,{ }^{1)}$

* Dr. Ir. J. Xu and Prof. Dr.-Ing. U. Hoffmann, Institut für Technische Chemie, TU Braunschweig, Hans-Sommer-Straße 10, D-3300 Braunschweig, and Dr.-Ing. Thomas Rickert, Rheinisch-Westfälischer TÜV, Steubenstraße 53, D-4300 Essen.

1) List of symbols at the end of the paper.

$$
G=\sum_{i=1}^{n} \sum_{j=1}^{n} \sigma_{\mathrm{i}, \mathrm{j}} \sum_{k=1}^{N}\left(r_{\mathrm{obs}, \mathrm{ik}}-r_{\mathrm{est}, \mathrm{ik}}\right)\left(r_{\mathrm{obs}, \mathrm{jk}}-r_{\mathrm{est}, \mathrm{jk}}\right)
$$

is often difficult and time comsuming, especially when the number of independent reactions, $n$, is large. This is often the case in petrochemical processes such as catalytic cracking, catalytic reforming, hydrocracking, desulphurization etc., in which reactants and products are numerous. If, for example, there are 30 independent reactions whose rates are fairly fast, 30 rate constants together with adsorption constants and rate constants of non-key reactions (if there are two or more paths linking any reactant with a product) have to be estimated. The minimization of the objective function, Eq. (1), has to be carried out by iterative search in a 30 plus dimensional space. This not only makes the parameter estimation extremely time consuming, but also renders the parameter estimates less reliable, since it is possible that there is more than one point at which all the partial derivatives are equal to or approaching zero. Since the classical method of model discrimination is implemented by re- 